\title{
Editorials
}

Ian Gilron MD FRCPC," Terence J. Coderre PhD ${ }^{\dagger}$

\section{Novel targets of pain modulation in anaesthesia: preventing painful memories}

I $\mathrm{N}$ the past decade, much has been learned about the neurophysiology of pain mechanisms and their modulation. A topic of great interest to both basic and clinical scientists is that of excitatory neurotransmitters including excitatory amino acids (EAAs) such as glutamate (GLU) which act at $\mathrm{N}$-methyl-D-aspartate (NMDA) receptors and neuropeptides such as substance $P(S P)$ which act at neurokinin (NK) receptors. ${ }^{1}$ In this issue of the Canadian Journal of Anaesthesia, Ishizaki et al. present an animal study of the "tail-clamp" MAC of isoflurane following intrathecal administration of the SP (NK-1) receptor antagonist CP96,345. ${ }^{2}$ Briefly, after receiving varying intrathecal doses of $\mathrm{CP} 96,345$, rats underwent MAC determination studies under isoflurane anaesthesia. MAC was subsequently remeasured after a "reversal" dose of SP. Results showed that CP96,345, in a dose-dependent fashion, reduced the MAC of isoflurane by $15.3 \%$ (at the highest dose of $100 \mu \mathrm{g}$ ) and furthermore that this MAC reduction was reversed by intrathecal SP. From this it was concluded that the pain transmitting activity of the NK-1 receptor is important in the determination of MAC for isoflurane. Simply put, by exerting an analgesic effect and thus diminishing noxious stimulation by the tail-clamp, CP96,345 produced a modest reduction in anaesthetic requirements. With the ongoing discovery of diverse mediators of nociception and analgesia, it is necessary to view such investigations in perspective so as to appreciate their potential relevance in clinical practice.

\section{Neurophysiology of pain transmission}

Transmission of noxious stimuli from peripheral nociceptors to the spinal cord through $A-\delta$ and $C$ nerve fibres involves the synaptic release of afferent neurotransmitters onto second order neurons in the dorsal horn of the spinal cord. Such transmitters are characterized by their production of excitatory postsynaptic potentials in dorsal horn neurons which may result in the transmission of pain signals to the brain. While this synaptic excitation is enhanced by several facilitatory mechanisms (referred to as central sensitization) it is also modulated by suppressive mechanisms including descending inhibitory pathways and segmental inhibitory interneurons. ${ }^{1}$ One of the objectives of studies such as that by Ishizaki $e t a l$. is to understand further the role of specific afferent transmitter systems by evaluating the effect of their agonism and antagonism.

\section{Afferent neurotransmitters of nociception}

Afferent neurotransmitters which have been of major interest in the field of pain include: 1) EAAs such as GLU and aspartate (ASP) and 2) neuropeptides such as tachykinins (e.g., SP), calcitonin gene-related peptide (CGRP), vasoactive intestinal polypeptide (VIP), somatostatin and bombesin (Figure). Postsynaptic receptors in the dorsal horn which bind EAAs include: i) N-methyl-Daspartate (NMDA), ii) 2-amino-3-hydroxy-5-methyl-4isoxazole-proprionic acid (AMPA), iii) kainate (KA) and iv) metabotropic glutamate receptors (mGluR). Excitatory amino acid stimulation of these receptors during in vivo experiments generally produces acute pain

\footnotetext{
"Departments of Anaesthesia and Neurology \& Neurosurgery, McGill University, Montreal

tPain Mechanisms Laboratory, Clinical Research Institute of Montreal, Canada.

Address correspondence to: Dr. Ian Gilron, Department of Anaesthesia, Room S5.05, Royal Victoria Hospital, 687 Pine Avenue West, Montreal, Quebec H3A 1 Al.
} 


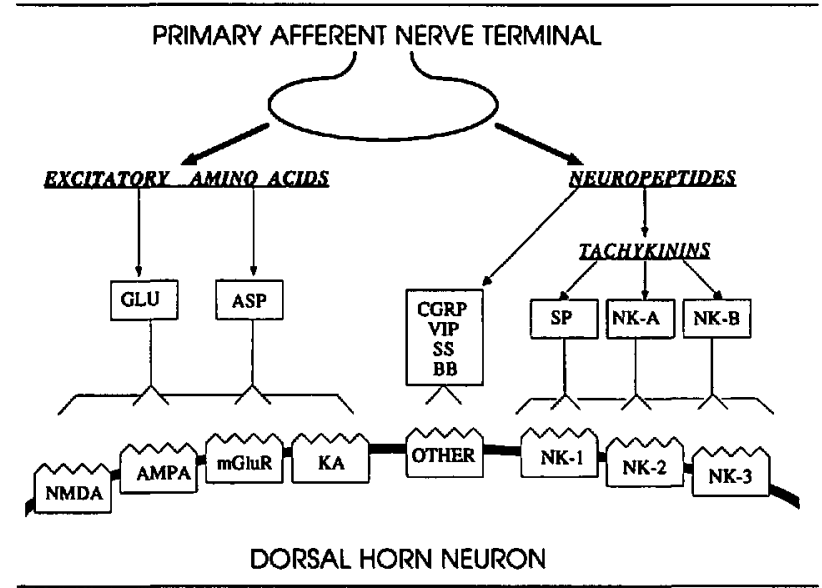

FIGURE This schematic illustrates the predominant neurochemical mediators of pain transmission between the terminal of a primary afferent nociceptive neuron and a second order spinal cord dorsal horn neuron.

Abbreviations:

GLU = glutamate;

$A S P=$ aspartate;

CGRP = calcitonin gene-related peptide;

VIP = vasoactive intestinal polypeptide;

SS = somatostatin;

$\mathrm{BB}=$ bombesin;

$\mathrm{SP}=$ substance $\mathrm{P}$;

NK-A, $B, 1,2,3=$ neurokinin-A, $B, 1,2,3$, respectively;

NMDA = N-methyl-D-aspartate;

AMPA = 2-amino-3-hydroxy-5-methyl-4-isoxazole-proprionic acid; $\mathrm{KA}=$ kainate; $\mathrm{mGluR}=$ metabotropic glutamate receptor.

behaviour or hyperalgesia while antagonism at these receptor sites blocks the development of hyperalgesia. ${ }^{1}$ The tachykinin group of neuropeptides include SP, NK-A and NK-B and bind to NK receptors (subtypes NK-1, NK-2 and NK-3) to produce pain behaviour and hyperalgesia which also can be blocked by specific antagonism. The tachykinins SP, NK-A and NK-B bind with high affinity to NK-1, NK-2 and NK-3 receptors, respectively, although some cross-interaction does exist. While other neuropeptides such as CGRP are known to be released in the dorsal horn by noxious afferent stimulation, ${ }^{3}$ functional antagonists for these peptide systems have yet to be discovered thus leaving in question the role of their receptors in nociception. ${ }^{1}$

\section{Substance $P$ and Neurokinin-1 (NK-1) Receptor Antagonism}

Substance $P$, first isolated in 1931 by von Euler and Gaddum, is an 11-amino acid neuropeptide which is a member of the family of related tachykinin or neurokinin peptides. ${ }^{4}$ Anatomical and physiological evidence which supports the role of SP in nociception includes its prominent expression in the substantia gelatinosa of the spinal cord dorsal horn, and the observation of its spinal release following noxious stimulation. ${ }^{5}$ While noxious stimulus- induced release of EAAs, such as GLU, produce rapid post-synaptic depolarizations, transmission by SP and other neuropeptides elicit slower and more persistent depolarization suggesting a functional difference between these two transmitter systems. ${ }^{1}$ Following the discovery of the novel potent non-peptide NK-1 antagonist CP-96,345, ${ }^{6}$ this agent was studied behaviourally and electrophysiologically for its antinociceptive effects in thermal, mechanical and chemical pain models. ${ }^{7,8}$ Since then, tens of studies have examined the antinociceptive effects of this agent. In a different approach, Ishizaki $e t a l$. have evaluated CP-96,345 by measuring its isoflurane-sparing effect in rats. While the use of this approach emphasizes the importance of afferent transmission and spinal cord-mediated responses in the determination of MAC, it can also be thought of as a measure of analgesia in the anaesthetized animal. Although this paradigm does not measure behavioural analgesic effect in the intact organism, it does measure the utility of this agent as part of a balanced anaesthetic regimen.

A conceptual shortcoming of the singular use of SP antagonists in preventing pain transmission is the presence of other systems (e.g., EAAs) which may continue to function despite the SP antagonism. In fact, it is interesting to note that SP and GLU are co-localized in common afferent nerve terminals. ${ }^{9}$ Together with evidence that co- administration of SP and GLU produces mutual augmentation of pain behaviours, the possibility of a functional interaction between SP and GLU suggests that antagonism of only one of these transmitters may be inadequate in blocking pain transmission. ${ }^{1}$

\section{Excitatory neurotransmitters and central sensitization}

Further appreciation of the value of specifically blocking pain-induced excitatory transmission may be gained by reviewing some basic aspects of central sensitization to pain. Basic and clinical evidence suggests that noxious peripheral stimulation produces central nervous system sensitization which subsequently influences pathological pain processes. ${ }^{10}$ Excitatory neurotransmitters are important participants in pain transmission and the subsequent development of central sensitization. Transmission of painful stimuli produces the release of SP and GLU. GLU acts post-synaptically on NMDA and metabotropic receptors while SP acts on NK receptors (NK-1 and 
NK-2) to produce long-lasting effects. In addition to producing prolonged cell depolarization, activation of NMDA and NK-1 receptors produces calcium $\left(\mathrm{Ca}^{++}\right.$ion) influx through NMDA receptor-operated and voltagegated calcium channels. The increased intracellular calcium is an important trigger which stimulates the production of various intracellular messengers, as well as proto-oncogenes such as c-fos and c-jun. Intracellular messengers are critically involved in the phosphorylation of various proteins, including those involved in the regulation of ion channels. The protein products of the proto-oncogenes $\mathrm{c}-$ fos and c-jun participate in the regulation of mRNA encoding dynorphin and enkephalin peptides in spinal cord, and influence long-term changes in cellular function. The metabolic activation which ensues from afferent-induced excitatory neurotransmission thus results in the development of hyperalgesia in both experimental and clinical models.

\section{Preventing painful memories}

In a recent animal study, Sun $e t a l$. demonstrated that halothane and/or nitrous oxide failed to suppress pain-induced spinal c-fos expression while fentanyl produced a $50 \%$ reduction in c-fos. ${ }^{11}$ In an accompanying editorial, Munglani $e t$ al. emphasize the point that a solely nitrous oxide/volatile-based anaesthetic leaves the spinal cord "unprotected" against painful stimulation despite the adequate prevention of subjective responses to stimulation. ${ }^{12}$ Although the clinical utility and methods of preemptive analgesic strategies continue to be questioned, ${ }^{13}$ perhaps the concept of preventing pain-induced sensitization should emerge as a perioperative anaesthetic goal to be included among those traditional elements such as amnesia, analgesia and reflex inhibition.

\section{Conclusion}

Issues of concern regarding the clinical utility of NK-1 antagonists such as CP96,345 include their inability to prevent transmission by other systems such as EAAs as well as possible non-specific side effects. ${ }^{14}$ For these reasons, it is difficult to speculate on the future role of such agents in the anaesthetist's pharmacopoeia. It is possible that clinically effective analgesia by antagonism of excitatory neurotransmitters will require the simultaneous blockade of $\mathrm{NK}, \mathrm{EAA}$ and perhaps even other as yet undiscovered receptor systems. The ongoing research and development of novel agents such as $\mathrm{SP}$ antagonists may provide opportunities to further diversify a balanced anaesthetic/analgesic approach and as well to develop strategies which prevent the long term consequences of pain.

\section{La redéfinition de nouvelles cibles en anesthésie : la prévention de la mémoire douloureuse}

Maintenant, on connaît mieux la neurophysiologie des mécanismes de la douleur et de leur modulation. La recherche fondamentale et clinique s'intéresse en effet beaucoup plus aux neurotransmetteurs chimiques dont les acides aminés excitateurs (EAA) comme le gluatamate (GLU) qui agit au niveau des récepteurs du N-méthyl-D-Aspartate (NMDA) et aux neuropeptides comme la substance $\mathrm{P}(\mathrm{SP})$ qui agit sur les récepteurs de la neurokinine (NK). ${ }^{1}$ Dans ce numéro du Journal canadien d'anesthésie, Ishisaki et al. présentent les résultats d'une étude réalisée sur le MAC de l'isoflurane déterminé par le clampage de la queue du rat après l'administration de l'antagoniste de SP (NK1) le CP96 $345 .{ }^{2}$ En bref, après l'administration de doses sous-arachnoïdiennes variables de CP96 345 à des rats, ces derniers ont été anesthésiés à l'isoflurane dont le $\mathrm{MAC}$ a été déterminé. Après une dose de neutralisation de SP, ils mesuraient ensuite le MAC de nouveau. Leurs résultats ont montré que le CP96 345 diminuait, proportionnellement à la dose, le MAC de l'isoflurane de $15,3 \%$ (pour la dose la plus élevée de $100 \mu \mathrm{g}$ ) et que subséquemment, cette réduction du MAC était annulée par l'administration sous-arachnoïdienne de SP. Pour simplifier, on peut dire qu'en exerçant un effet analgésique et donc en diminuant la nocivité du clampage de la queue, le CP96 345 réduisait le besoin d'anesthésique de façon modeste. Avec la découverte continuelle de nouveaux médiateurs de la nociception et de l'analgésie, il devient nécessaire de reconsidérer les résultats de ces recherches sous l'angle de leur pertinence en clinique.

La neurophysiologie de la transmission de la douleur La transmission des stimuli nocifs, des nocicepteurs périphériques vers la moelle épinière par les fibres nerveuses $\mathrm{A}$ - et $\mathrm{C}$, nécessite la libération au niveau synaptique de neurotransmetteurs afférents dans les neurones de classe deux de la corne dorsale de la moelle épinière. Ces neurotransmetteurs produisent 


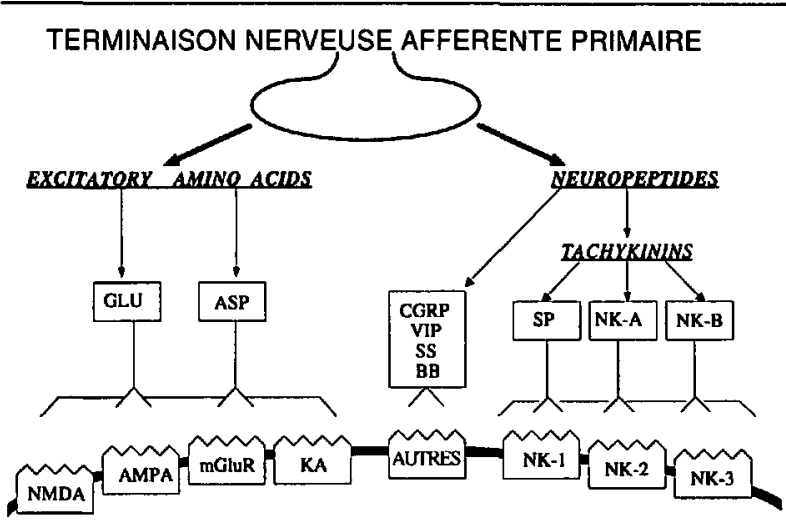

NEURONE DE LA CORNE DORSALE

FIGURE Ce schéma illustre les principaux médiateurs neurochimiques de la transmission douloureuse entre la terminaison d'un neurone primaire nociceptif afférent et un neurone de deuxième classe de la corne dorsale de la moelle.

Abréviations:

$\mathrm{GLU}=$ glutamate

$\mathrm{ASP}=$ aspartate,

CGRP = peptide génique calcitonique,

VIP $=$ polypeptide intestinal vasoactif,

$\mathrm{SS}=$ somatostatine,

$\mathrm{BB}=$ bombésine,

$\mathrm{SP}=$ substance $\mathrm{P}$,

NK-A, $B, 1,2,3$ = neurokine-A,B, 1, 2,3,

NMDA $=\mathrm{N}$-méthyl-D-aspartate,

AMPA = acide 2-amino-3hydroxy-5-méthyl-4-isoxasole-propionique, $\mathrm{KA}=$ kainate, $\mathrm{mGLUr}=$ récepteur métabotropique glutamate .

des potentiels d'excitation spécifiques au niveau des neurones de la corne dorsale responsables de la transmission de la douleur au cerveau. Alors que l'excitation synaptique est amplifiée par plusieurs mécanismes facilitateurs (la sensibilisation centrale), elle est aussi modulée par des mécanismes suppresseurs dont les voies de conduction inhibitrices descendantes et les interneurones inhibiteurs segmentaires. ${ }^{1}$ L'étude de Ishizaki et al. visait, entre autres, à mieux faire comprendre le rôle des systèmes de la transmission afférente par l'évaluation des effets de leur agonisme et de leur antagonisme.

Les neurotransmetteurs afférents de la nociception Dans le domaine de la douleur, on s'intéresse principalement 1) aux EAA comme GLU et l'aspartate (ASP) et 2) aux neuropeptides comme les tachykinines (par ex, $\mathrm{SP}$ ), le peptide génique calcitonine (CGRP), le polypeptide intestinal vasoactif (VIP), la somatostatine et la bombésine (Figure). Parmi les récepteurs postsynaptiques de la corne dorsale qui se lient aux EAA on compte : i) le N-méthyl-D-aspartate (NMDA), ii) l'acide 2-amino-3hydroxy-5-méthyl-4-isoxasole-propionique
(AMPA), iii) le kainate (KA) et iv) les récepteurs du glutamate métabotropique (mGluR). La stimulation de ces récepteurs par les acides aminés excitateurs pendant des expériences in vivo provoque une attitude douloureuse aiguë ou de l'hyperalgie alors que l'antagonisme de ces sites récepteurs bloque les manifestations de l'hyperalgie. ${ }^{1}$ Le groupe tachykinine des neuropeptides dont $S P$, NK-A et NK-B se lient aux récepteurs NK (sous-types NK-1, NK-2 et NK-3) par la suite pour produire l'attitude douloureuse et l'hyperalgie sujets au blocage par des antagonistes spécifiques. Les tachykinikes SP, NK-A et NK-B se lient respectivement avec beaucoup d'affinité aux récepteurs NK-1, NK-2 et NK-3, mais ces liaisons peuvent se croiser. Alors que d'autres neuropeptides comme CGRP sont vraisemblablement libérés dans le corne dorsale par la stimulation nocive afférente, ${ }^{3}$ on ne connait pas encore les antagonistes fonctionnels de ces systèmes peptidiques, ce qui laisse sans réponse la question du rôle de leurs récepteurs sur la nociception. ${ }^{1}$

La substance $P(S P)$ et l'antagonisme du récepteur neurokinine-1 (NK-1)

Isolée en 1931 par von Euler et Gaddum, la substance $P$ est un acide 11 -amino neuropeptidique membre de la famille des peptides apparentée à la tachykinine et à la neurokinine. ${ }^{4}$ Le rôle de SP dans la nociception est supporté anatomiquement et physiologiquement par son apparition dans la substance gélatineuse de la corne dorsale et l'observation de sa libération dans la moelle épinière après une stimulation nocive. ${ }^{5}$ Tandis que la libération provoquée par les stimuli nocifs des EAA comme GLU, provoque des dépolarisations postsynaptiques rapides, la transmission par SP et d'autres neuropeptides entraîne une dépolarisation plus lente et plus prolongée suggérant ainsi une différence fonctionnelle entre ces deux transmetteurs. ${ }^{1}$ Récemment découvert, l'antagoniste puissant non peptidique de $\mathrm{NK}-1$, le CP96 346, ${ }^{6}$ a subi une analyse comportementale et électrophysiologique en rapport avec ses effets antinociceptifs sur des modèles de douleur thermique, mécanique et chimique., ${ }^{78}$ Depuis, des dizaines d'études portant sur les propriétés antinociceptives de cet agent ont suivi. Utilisant une approche différente, Ishizaki et al. ont évalué le CP96 346 en mesurant son effet d'épargne sur l'isoflurane chez le rat. Cette approche met en évidence l'importance de la transmission afférente et des réponses à médiation spinale sur la façon de déterminer le MAC mais elle peut être aussi considérée comme une mesure de l'analgésie chez l'animal anesthésié. Bien que ce paramètre ne mesure pas l'effet analgésique comportemental dans un organisme intact, il mesure la contribution de l'isoflurane comme composante d'une anesthésie équilibrée. 
Le concept même de l'utilisation d'antagonistes de SP pour la prévention de la transmission de la douleur est limité par la présence d'autres systèmes (par ex., les EAA) qui peuvent continuer de fonctionner malgré l'antagonisme de SP. En effet, il est intéressant de noter que SP et GLU sont situés sur des terminaisons nerveuses afférentes communes. ${ }^{9}$ À l'évidence que l'administration conjointe de SP et de GLU augmente avec réciprocité les comportements douloureux, s'ajoute la possibilité d'une interaction fonctionnelle entre SP et GLU, ce qui porte à croire que l'antagonisme d'un seul de ces transmetteurs peut être insuffisante pour bloquer la transmission de la douleur.

\section{Neurotransmetteurs excitateurs et sensibilisation centrale}

Le rappel de certains aspects fondamentaux de la sensibilisation centrale à la douleur nous permettra de mieux comprendre le rôle de la transmission excitatrice sur le blocage de la douleur. Certains résultats de recherche clinique et fondamentale suggèrent que la stimulation périphérique nocive sensibilise le système nerveux central et agit ainsi sur les processus pathologiques de la douleur. ${ }^{10}$ Les neurotransmetteurs de l'excitation facilitent la transmission de la douleur et le phénomène de sensibilisation centrale subséquente. La transmission des stimuli douloureux entraîne la libération de $S P$ et de GLU. L'activité de GLU sur NMDA et les récepteurs métabotropiques s'effectue au niveau postsynaptique alors que SP agit sur les récepteurs NK (NK-1 et NK-2) pour produire des effets prolongés. En plus d'enclencher une dépolarisation cellulaire prolongée, l'activation des récepteurs NMDA et NK-l provoque un influx de calcium (l'ion $\mathrm{Ca}^{++}$) à travers les récepteurs contrôlés par NMDA et les canaux calciques éclusés par le voltage. Le calcium intracellulaire est un important facteur déclenchant de la production de différents messagers intracellulaires, aussi bien des proto-oncogènes comme le c-fos et le c-jun. Les messagers intracellulaires sont essentiels à la phosphorylation de plusieurs protéines dont celles qui sont engagées dans la régulation des canaux ioniques. Les dérivés protéiniques des proto-oncogènes cfos et c-jun participent à la régulation par encodage mRNA de la dynorphine et des encéphalines peptidiques dans la moelle épinière et influencent les modifications à long terme de la fonction cellulaire. L'activation métabolique subséquente à la neurotransmission excitatrice à partir des afférents provoque donc une hyperalgie tant expérimentale que clinique.

\section{La prévention de la mémoire douloureuse}

Dans un travail publié récemment, Sun et al. ont montré que l'halothane et/ou le protoxyde d'azote sont incapables arrêter la production induite par la douleur de c-fos médullaire alors que le fentanyl la réduit le c-fos de $50 \%{ }^{11}$ Dans l'éditorial qui accompagnait l'article, Munglani $e t$ al. soulignaient le fait que l'anesthésic au protoxyde d'azote seul ne protégeait pas la moelle épinière contre la stimulation douloureuse malgré une protection subjective adéquate. ${ }^{12}$ Bien que l'efficacité et les moyens de réaliser la coanalgésie sont toujours mis en doute, ${ }^{13}$ le concept de la prévention analgésique devrait peut-être être proposé comme un objectif anesthésique avec les éléments traditionnels comme l'amnésie, l'analgésie et l'inhibition des réflexes.

\section{Conclusion}

La question de l'utilité clinique des antagonistes NK-1 comme le CP96 345 doit tenir compte du fait qu'ils sont impuissants à prévenir la transmission de la douleur par les autres systèmes comme les EAA et de leurs effets secondaires non spécifiques. ${ }^{14}$ Pour ces raisons, on peut difficilement spéculer sur la place éventuelle de ces agents dans la pharmacopée de l'anesthésiste. Il est possible qu'une analgésie efficace par antagonisme des neurotransmetteurs excitateurs nécessite le blocage simultané de NK, EAA et peut-être même d'autres systèmes de récepteurs non identifiés présentement. La recherche et le développement actuels de nouveaux agents comme les antagonistes SP permettront peut-être de diversifier encore plus les méthodes de l'anesthésie et de l'analgésie équilibrées. Des stratégies capables de prévenir les conséquences à long terme de la douleur pourraient aussi être élaborées.

\section{References}

1 Taksh TL, Malmberg AB. Central pharmacology of nociceptive transmission. In: Wall PD, Melzack $\mathrm{R}$ (Eds.). Textbook of Pain, 3rd ed. London: Churchill Livingstone Inc., 1994: 165-200.

2 Ishizaki K, Karasawa S, Takahashi K, Hasegawa $M$, Goto $F$. Intrathecal neurokinin-1 receptor antagonist reduces isoflurane MAC in rats. Can J Anaesth 1997; 44: 543-549.

$3 \operatorname{Kar} S, R e e s R G$, Quirion $R$. Altered calcitonin generelated peptide, substance $P$ and enkephalin immunoreactivities and receptor binding sites in the dorsal spinal cord of the polyarthritic rat. Eur J Neurosci 1994; 6: 345-54.

4 Cooper JR, Bloom FE, Roth RH. The Biochemical Basis of Neuropharmacology, 5 th ed. New York: Oxford University Press, 1986: 363-5.

5 Go VLW, Yaksh TL. Release of substance P from the cat spinal cord. J Physiol 1987; 391: 141-67.

6 Snider RM, Constantine JW, Lowe JA III, et al. A potent nonpeptide antagonist of the substance $P\left(\mathrm{NK}_{1}\right)$ receptor. Science 1991; 251: 435-7. 
7 ramamoto $T$, Yaksh TL. Stereospecific effects of a nonpeptidic NKI selective antagonist, $\mathrm{CP}-96,345$ : antinociception in the absence of motor dysfunction. Life Sci 1991; 49: 1955-63.

8 Radhakrishnan V, Henry $J$. Novel substance $P$ antagonist, CP-96,345, blocks responses of cat spinal dorsal horn neurons to noxious cutaneous stimulation and to substance P. Neurosci Lett 1991; 132: 39-43.

9 De Biasi S, Rustioni $A$. Glutamate and substance P coexist in primary afferent terminals in the superficial laminae of spinal cord. Proc Nat Acad Sci 1988; 85: 7820-4.

10 Coderre T], Katz J, Vaccarino AL, Melzack $R$.

Contribution of central neuroplasticity to pathological pain: review of clinical and experimental evidence. Pain 1993; 52: 259-85.

11 Sun WZ, Shyu BC, Shieh JY. Nitrous oxide or halothane, or both, fail to suppress $c$-fos expression in rat spinal cord dorsal horn neurones after subcutaneous formalin. Br J Anaesth 1996; 76: 99-105.

12 Munglani R, Fleming BG, Hunt SP. Remembrance of times past: the significance of $\mathrm{c}$-fos in pain (Editorial). Br J Anaesth 1996; 76: 1-4.

13 Woolf $C J$, Chong $M-S$. Preemptive analgesia - treating postoperative pain by preventing the establishment of central sensitization. Anesth Analg 1993; 77: 362-79.

14 Hill $R G$. Discovery of new analgesic drugs: issues relating to centrally acting agents given systemically. In: Campbell JN (Ed.). Pain 1996 - An updated review. Seattle: IASP Press, 1996: 377-80. 